\title{
Hurst Exponent-Based Nonlinear Analysis for the Identification of Arrhythmia-Affected Cardiac Systems
}

Chiranjit Maji ( $\sim$ chiranjit.nitdphysics@yahoo.in )

National Institute of Technology Durgapur https://orcid.org/0000-0001-9324-7923

\section{Pratyay Sengupta}

Indian Institute of Technology Madras

Anandi Batabyal

Florida State University

Hirok Chaudhuri

NIT-Durgapur: National Institute of Technology Durgapur

\section{Research Article}

Keywords: Arrhythmia, Cardiac disorders, ECG analysis, Nonlinear analysis, Empirical Mode Decomposition, Hurst exponent

Posted Date: October 20th, 2021

DOI: https://doi.org/10.21203/rs.3.rs-898132/v1

License: (9) (1) This work is licensed under a Creative Commons Attribution 4.0 International License. Read Full License 


\title{
Hurst Exponent-Based Nonlinear Analysis for the Identification of Arrhythmia-Affected Cardiac Systems
}

\author{
Chiranjit Maji ${ }^{\mathrm{a}}$, Pratyay Sengupta ${ }^{\mathrm{b}, \mathrm{c}}$, Anandi Batabyal ${ }^{\mathrm{b}, \mathrm{d}}$, Hirok Chaudhuri $^{\mathrm{a}}$

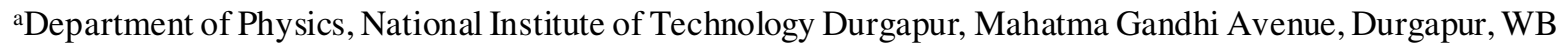 \\ 713209, India \\ bDepartment of Biotechnology, National Institute of Technology Durgapur, Mahatma Gandhi Avenue, Durgapur, \\ WB 713209, India \\ ${ }^{c}$ Current Address: Department of Biotechnology, Bhupat and Jyoti Mehta School of Biological Sciences, Indian \\ Institute of Technology Madras, Chennai, TN 600036, India \\ ${ }^{\mathrm{d} C u r r e n t}$ Address: Department of Biological Science, Cell and Molecular Biology, Florida State University, 319 \\ Stadium Dr, Tallahassee, FL 32304, United States of America \\ *Correspondence to be addressed: chiranjit.nitdphysics@yahoo.in (C.M)
}

\begin{abstract}
The stochastic nature of the human heart, a complex biological system, is evident from electrocardiogram (ECG) signals, which are weak, non-linear and non-stationary signals. These temporal variations of electromagnetic pulses emanated from the heart are instrumental in indicating the cardiac health. The Empirical Mode Decomposition (EMD) technique was employed in order to decompose a total of 64 ECG signal data of arrhythmic and normal subjects, obtained from widely used MIT-BIH databases, into a finite number of Intrinsic Mode Functions (IMFs). The rationale behind using this strategy was to extract non-linear features of ECG signals which are not explicitly expressed, while keeping the original signal unaltered. Following removal of non-stationary noises from the ECG signals by the Savitzky-Golay (SG) filter, popular non-linear parameter Hurst Exponent (H) was estimated for every IMF by employing the R/S technique. A distinct difference between $\mathrm{H}$ values of 1 st IMFs between normal individuals and arrhythmia affected patients was identified. This observation was further validated through an age-based and gender-based analysis, which demonstrated a unique alteration pattern with age. The study showed $94.92 \%$ probability in detection of arrhythmia in a patient. Adopting this EMD-based procedure for ECG data analysis and disease prediction may assist in reducing our dependence on intuition-based diagnosis of ECG reports by medical practitioners and may provide novel insights into the functioning of the human heart which might help develop new biomedical strategies to combat cardiac disorders.
\end{abstract}

Keywords: Arrhythmia; Cardiac disorders; ECG analysis; Nonlinear analysis; Empirical Mode Decomposition; Hurst exponent 


\section{Introduction}

Recent times have witnessed a surge in occurrence of cardiovascular diseases. Arrhythmia is one of the notable heart ailments having symptoms including anxiety, palpitations at rest and exertion, reduced physic al ability and breathlessness [1]. The occurrence of arrhythmia is also associated with the onset of other severe complications like stroke and heart failure. Electrocardiogram (ECG) signals are conventional and well-established tools for cardiologists to assess the health of the heart in an easy and cost-effective manner [2]. Modern techniques such as the Holter test, echocardiogram and stress tests are also used for the diagnosis of a cardiac patient but are sophisticated and relatively expensive, thus limiting accessibility of the less-privileged populations of developing nations to these techniques [3]. However, conventional ECG analysis is typically based on identification of temporal variations in the signal, such as its wavelength and amplitude. Thus, manual interpretation by the cardiologists sometimes fails to provide certain vital information about the heart by conventional ECG-based analysis alone [4]. Often time is a constraint for interpretation of ECG reports of critical patients suffering from emergency medical issues, which necessitates analysis of these reports automatically [5]. Therefore, a computerized interpretation of medical data like electrocardiogram has been of interest to researchers over the last few decades. Efforts have been made so far in the identification of cardiac abnormalities such as premature ventricular complexes, atrial fibrillation etc. by taking into consideration different components of the ECG signals and analysing them with respect to ECG signals emanated from normally functioning cardiac systems [6-8]. However, studies involving characterization of ECG signals using nonlinear approaches are relatively rare.

Diverse non-linear techniques that involve a shift to the frequency domain from the time domain, such as multifractal analysis, wavelet transform, recurrence etc. are often used in physiology and medical sciences $[9,10]$. Empirical Mode Decomposition (EMD), which seems to be an attractive tool for extraction of nonlinear features from biomedical signals, essentially involves decomposition of the signal into a finite number of Intrinsic Mode Functions (IMFs), keeping its original properties intact [11]. This multi-scale analysis method, which is used widely for the prediction of the signal's trend, does not rely on any prior knowledge [12,13]. A nonlinear parameter, Hurst exponent $(\mathrm{H})$, which is crucial for elucidating the regularity and scaling in a time series, might serve as an effective parameter for interpretation of the underlying dynamics of the nonlinear cardi ac system and for the characterization of arrhythmic patients $[14,15]$.

PhysioNet is an open access collection of physiological signals and clinical data besides related open-source software. In this study, we collected publicly available ECG signals and proposed a pipeline which will help to extract the nonlinear features buried in ECG signals by emphasizing on the nonlinear topographies of IMFs, for comprehensive analysis of ECG reports. We sought to distinguish arrhythmia affected patients from the normal human subjects based on a comparative study of Hurst exponents of a particular IMF retrieved from respective ECG signals, which can prove to be a potential strategy for identification of arrhythmia and subsequently, may assist to establish a new paradigm in cardiology. 


\section{Materials and methods}

\section{Data Collection and Preprocessing}

Full-length ECG time series data of 48 arrhythmic patients were downloaded from popular MIT-BIH Arrhythmia Database (https://physionet.org/content/mitdb/1.0.0/) [16,17]. The patients were 25 men aged 32 to 89 years, and 22 women aged 23 to 89 years. However, records 201 and 202 were taken from the same male subject. Full-lengthECG time series data of 18 normal subjects were collected from MIT-BIH Normal Sinus Rhythm Database (https://www.physionet.org/content/nsrdb/1.0.0/). Normal subjects include 5 men aged 26 to 45, and 13 women aged 20 to 50 having no significant arrhythmias. Each ECG data was recorded for the time duration of 60 seconds. The Savitzky-Golay (SG) filter was employed for pre-processing of ECG signals in order to maximize noise reduction with minimal signal distortion [18, 19]. The SG filter was performed by the MATLAB v.R2016a (https://in.mathworks.com/).

\section{Empirical Mode Decomposition Analysis of the ECG Signals}

Empirical Mode Decomposition (EMD) technique was applied to each of the filtered ECG signals, decomposing them into a finite number of Intrinsic Mode Functions (IMFs) [20]. To retrieve the maximum number of IMFs, the number of iterations was set to 150 . The algorithm is described as follows:

1. $x(t)$ is considered as a signal and the initiation of the sifting process is started by identification of all the local maxima and minima of

2. Then all the local maxima are to be connected by a cubic spline curve using interpolation, said as upper envelope $e_{u}(t)$. Similarly, the local minima are connected to get the lower envelope $e_{l}(t)$.

3. The mean of the upper envelope and the lower envelope is calculated and denoted by $m_{l}(t)$, i.e.,

$$
m_{l}(t)=\frac{\left[e_{u}(t)+e_{l}(t)\right]}{2}
$$

4. The mean is then subtracted from the main signal $x(t)$ to get the first proto-IMF $h_{1}(t)$. Thus,

$$
h_{1}(t)=x(t)-m_{1}(t)
$$

5. Due to multiple extrema present in between two consecutive zero crossings, the sifting process is to be applied continuously to $h_{k}(t)$, the kth proto-IMF. Once, this satisfies the IMF conditions, the first IMF $c_{l}(t)$ is obtained.

6. The sifting process gets stopped once reaching the stopping criterion which is characterized by the Sum of Deviations (SD) as 


$$
S D=\sum_{t=0}^{T} \frac{\left[h_{k-1}(t)-h_{k}(t)\right]^{2}}{h_{k-1}(t)^{2}}
$$

The first IMF $c_{1}(t)$ is obtained when the SD is smaller than or equal to the threshold value $S D_{\max }$. The typical values of $S D_{\max }$ lies between 0.2 and 0.3 .

7. After that, the IMF is deduced from the original signal to find the first residual signal $r_{1}(t)$ by

$$
r_{1}(t)=x_{1}(t)-c_{1}(t)
$$

8. This residual signal is considered as the original signal to produce further a pair of IMF and residual signal. The procedure is allowed to continue until the $\mathrm{N}^{\text {th }}$ residue $r_{N}(t)$ turns to be a constant or with a single extremum or having a monotonic slope.

9.Finally, combining all the above steps, the original signal can be expressed as

$$
x(t)=\sum_{n=1}^{N} c_{n}(t)+r_{N}(t)
$$

Each IMF should satisfy the following characteristics [21]:

A.The number of zero-crossing must be equal or differ by one unit to the number of extrema (assuming it has at least two extrema).

B.Each IMF should be symmetrical with respect to the local mean.

The above mentioned EMD processes were performed by the MATLAB v.R2016a (https://in.mathworks.com/).

\section{Statistical Significance of Intrinsic Mode Functions}

The correlation coefficient between the IMFs and the original signal were calculated to identify the IMFs with the real components of the original signal $[22,23]$. The correlation coefficients $\left(C_{x c_{N}}\right)$ were calculated using the following equations $[24,25]$ :

$$
C_{x c_{N}}=\frac{\int x(t) c_{n}(t)}{\sqrt{\int x^{2}(t) d t \int c_{n}^{2}(t) d t}}
$$

A hard threshold $\lambda$ is used to select the significant IMFs described as

$$
\lambda=\frac{\max \left(C_{x c_{N}}\right)}{\eta}
$$

Where, $\eta$ is a ratio factor greater than 1.0 [23]. In this study, IMFs with $C_{x c_{N}} \geq \lambda$ were considered to be significant. 


\section{Estimation of Hurst Exponents}

The Hurst exponent $(H)$ was calculated for each IMF by R/S technique, proposed by Mandelbrot and Wallis in 1969 [26]. The steps to get H using R/S method are described below:

1. The analysis begins by dividing the IMFs of length $L$ into d sub series $\left(Z_{i, m}\right)$ of length $n$.

2. The sample mean $\left(E_{m}\right)$ and the standard deviation $\left(S_{m}\right)$ are calculated for each sub-series, $m=1, \ldots ., d$

3. Then the sub-series data $\left(Z_{i, m}\right)$ is normalised as

$$
X_{i, m}=Z_{i, m}-E_{m} \quad \text { for } i=1, \ldots ., n . \quad \quad .
$$

4. A cumulative time series is created as

$$
Y_{i, m}=\sum_{j=1}^{i} X_{i, m} \quad \text { for } i=1, \ldots, n .
$$

5. The range is calculated from the cumulative series as

$$
R_{m}=\max \left\{Y_{l, m}, \ldots, Y_{n, m}\right\}-\min \left\{Y_{l, m}-Y_{n, m}\right\}
$$

6. The range is then re-scaled by dividing by $S_{m}$.

7. Finally, for all the sub-series of length $n$, the mean value of re-scaled range is considered as follow

$$
\left(\frac{R}{S}\right)_{n}=\frac{1}{d} \sum_{m}^{M}=1 \frac{R_{m}}{S_{m}}
$$

8. R/S statistics asymptotically follows the relation $\left(\frac{R}{S}\right)_{n} \approx c n^{H}$. The value of $\mathrm{H}$ can be evaluated by doing simple linear regression with the help of the equation

$$
\log \left(\frac{R}{S}\right)_{n}=\log C+H \log n
$$

\section{Statistical Analysis}

All the computation and statistical analyses were performed using in-house MATLAB v.R2016a (https://in.mathworks.com/) codes and GraphPad Prism v.7 (GraphPad Software, La Jolla California USA, www.graphpad.com). Graph plots were generated using MATLAB software and Adobe Illustrator. The whole methodology is followed by the schematic diagram, as shown in Figure. 1. 


\section{Results}

\section{Observatory results distinguish arrhythmia affected ECG signals}

The disease data and the normal data collected from the MIT-BIH databases were recorded for a time duration of 60 seconds. 21600 data points were observed for disease data series with a frequency of $360.01 \mathrm{sec}^{-1}$ while 7680 data points were observed for normal data series with a frequency of $128 \mathrm{sec}^{-1}$. Amplitudes for such data sets varied between -3.105 and $2.8 \mathrm{mV}$ for the arrhythmic patients while it ranged from -2.215 to $3.675 \mathrm{mV}$ in case of normal individuals. The temporal variation of the filtered data sets of one normal person (Patient ID: 19090) and one diseased patient (Patient ID: 100) are plotted in Figure 2. An observatory result shows that the normal ECG contains 47 peaks in 60 seconds, whereas 74 peaks are present in that of the disease data in the same interval. Furthermore, every cycle (wavelength) is repeated similarly in the normal ECG data, but that is not observed in the disease data sets. So, for further analysis an EMD operation was performed to each of the filter-smoothened data series (total 64 series, Supplementary Table S1) following the sifting process as described earlier. The number of IMFs retrieved from all the series varies between 10 and 14. The IMFs are also formed as a new time series with the data points equal to that of the original time series. The time series of IMFs corresponding to one disease data (Patient ID: 100) was plotted in Figure 3.

\section{Pattern recognition for the arrhythmia disease based on $\mathrm{H}$ value}

$\mathrm{H}$ of all the IMFs of all the 64 time series (including the diseased as well as normal patient, Supplementary Table S2) were evaluated to enumerate regularity and the scaling in the data series. R/S plots of all the IMFs (Patient ID: 100) are presented in Figure 4, depicting their corresponding $\mathrm{H}$ values. Comparison of $\mathrm{H}$ of different IMFs for a normal (Patient ID: 19090) and a disease (Patient ID: 100) data are presented in Table 1. The 1st IMF of each series is considered significant since $\mathrm{H}$ of those of the disease data significantly differ from those of the normal data. The $\mathrm{H}$ of all the 1st IMFs of all the time series were considered for further study and were listed in Table 2. The two-tailed student's t-test between the H of all 1 st IMFs corresponding to the disease data and normal data provided significant p-value of $0.3 \times 10^{-9}(<0.001)$ (Figure 5). The $\mathrm{H}$ values corresponding to normal patients range from 0.7886 to 0.9613 (mean $=0.8728$ and median $=0.8816)$ whereas the same for the diseased patients fluctuates in between 0.8685 to $1.0324($ mean $=0.9940$ and median $=1.0018)$.

\section{Selection of significant IMFs}

As the signature of the arrhythmia with respect to the $\mathrm{H}$ was observed in case of the $1 \mathrm{st}$ IMFs, thus each such series was examined to determine whether that was significant or not with the help of the equation no. 6 and 7 mentioned in Methods. The correlation coefficients were calculated for all IMFs of ECG signals (Supplementary Table S3). 1 st IMFs corresponding to the patient IDs 116, 122, 210, 219 and 223 (diseased patients) were observed to be insignificant and were eliminated from further analysis. 


\section{Age and gender-based subgrouping for pattern reconfirmation}

An intensive analysis was carried out on the $\mathrm{H}$ on the basis of age and gender. The values of $\mathrm{H}$ for the age based subgroups were following the similar trend as observed for the whole data set (Table 3). The values of $\mathrm{H}$ of the patients aged below 30 corresponding to the diseased and normal subjects exhibit mean values of 1.0013 and 0.8552 respectively. The mean values of $\mathrm{H}$ of diseased and normal cases for the individuals having age between 30 and 50 are evaluated as 0.9965 and 0.8809 consecutively. The same for the individuals with age ranging from 50 to 70 are 1.0026 and 0.8644 respectively. The assessment on the same platform for the aged patients (> 70 years) is skipped due to lack of normal subjects' corresponding data for that age boundary. The Hurst exponent values of the 1st IMFs of the gender-based subgroups were significantly $(\mathrm{p}<0.001)$ varied in case of male and female patients (Table 4). The mean of the $\mathrm{H}$ of the diseased male patients is 0.9992 , whereas the same for the normal male subjects is 0.8816 . For females, 0.9950 and 0.8547 are the mean values of the $\mathrm{H}$ corresponding to the diseased and normal individuals respectively. Also, the p-values for the same are listed in the above-mentioned tables.

\section{Discussion}

Alteration in heart rate occurs due to systole and diastole, induced by the actions of SA node, AV node as well as the His-Purkinje system [35]. Dysregulation of these mechanisms leads to various cardiovascular diseases, including arrhythmia [36,37]. Presence of such cardiovascular diseases is usually identified by an observation of the ECG report of an individual, which is mainly the graphical representation of the electrical potential of the heart [38]. Here Figure 2 reflects the distinction between a normal and diseased individual's electrocardiogram. Values of the maximum (and minimum) amplitude of all cycles are nearly constant throughout the measurement time for the normal data. However, a rapid variation on the same is observed in the ECG of disease data. Therefore, from the primary observation on the ECG plot in Figure 2, one can understand the deviation of the signal pattern for a diseased individual from that of a normal person. However, neither does such a representation quantify the seriousness $n$ or the type of the disease. The diagnosis of most of the cardiac disorders therefore continues to be largely dependent on intuitive analysis of the medical practitioner based on ECG reports. Interpretation of such reports may vary from one physician to another and may lead to inappropriate treatment by inexperienced physicians. Thus, owing to the advancement made in the domain of biophysics, quality of diagnosis of cardiovascular diseases through ECG signals could be improved by mounting on non-linear statistics and machine learning approaches [40 - 42].

EMD is chosen over other well-known nonlinear data analysis methods because it is a data- driven mechanism whereas other methods, like Fourier and wavelet-based methods, need some predefined basis functions for representing a signal $[43,44]$. Here, we have evaluated the IMFs of each data series by calculating the Hurst exponent of every IMF. The components of a signal i.e. the IMFs will provide deep insights aboutorigin of the disease, which prompted us to characterise the ECG signals by critically analysing the features of IMFs rather than the original signals. By omitting the $\mathrm{H}$ values for the patient IDs 19093, 19140 and 232 from our analysis, we found the $\mathrm{H}$ of 1st

IMFs to be ranging from 0.7886 to 0.9297 (mean $=0.8631$, median $=0.8705$ ) for normal persons and from 0.9361 to 1.0324 (mean $=0.9972$, median $=1.0025)$ for patients suffering from arrhythmia, which significantly differ from those of the normal data (Figure 5). The fact implies that the dynamic behaviour of the cardiac system of a normal person 
differs noticeably from the same of an arrhythmia affected individual. Therefore, the statement is true for 56 -time series data except 03 , i.e., there is a probability of $94.92 \%$ towards the predictability of arrhythmia disease in a patient by this analysis procedure.

The experimental result can also be enlightened with thehelp of the persons' gender and age- based grouping. Here, such distribution will lead us to assess whether the Hvalues (of 1st IMFs only) are evenly distributed throughout the range and also obey the similar pattern in short-range classification. The mean of the $\mathrm{H}$ of the diseased male and female patients were significantly distinct from the mean of the normal subjects as shown using box an $d$ whisker plot in Figure 6B. The discrete margin between the $\mathrm{H}$ of normal and diseased persons are also consistent with the genderbased grouping. Moreover, Table 2 portrays the significant distinction between $\mathrm{H}$ values corresponding to the different age groups of the diseased and normal subjects, which validates the assessment made earlier(Figure 6A). However, all the 1 st IMFs fall in the category of persistence type behaviour as $\mathrm{H}$ values of the same are always greater than 0.5. However, all the $\mathrm{H}$ values for the disease data $(\sim 1)$ are greater than that of the normal data. This observation suggests that the series of 1 st IMFs obtained from the disease data exhibits a stronger trend than that of the normal data [39]. So, the Hurst exponent can be a potential nonlinear factor for accurate identification of arrhythmia-affected cardiac systems in humans.

\section{Conclusion}

Signal analysis by nonlinear techniques has varied applications in understanding crucial physiological processes and in devising new biomedical strategies for disease prediction and treatment. This study highlights that the EMD can be a powerful tool for extraction of nonlinear features from electrocardiographic data. Our proposed pipeline demonstrated $94.92 \%$ probability towards identification of arrhythmia-affected cardiac systems in a patient. We showed that Hurst exponent might serve as a potential indicator of the health of the human heart and thereby can help in detection of cardiac arrhythmias owing to perturbations in the electrical activity of the heart.

Acknowledgement: The authors are indebted to the National Institute of Technology Durgapur for providing institutional support for carrying out the research activities. The authors would like to acknowledge Mr. Saroj Khutia, Ms. Kankana Seal and Ms. Suvashree Majumder for their sincere help to prepare the manuscript.

Declaration: Not applicable

Funding: No financial funding was required.

Conflicts of interest/Competing interests: The authors declare no conflict of interest.

Availability of data and material: The raw data was collected from the publicly available data respiratory of PhysioNet (https://physionet.org/content/mitdb/1.0.0/, https://www.physionet.org/content/nsrdb/1.0.0/).

Code availability: Not Applicable 


\section{Authors' contributions:}

Conceptualization: C.M., and, H.C.; Methodology: C.M., P.S., and, A.B.; Software: C.M., P.S., and, A.B.; Formal analysis: C.M.,P.S., A.B., and,H.C.; Investigation: C.M.,P.S., A.B., and,H.C.; Resources: H.C.; Data curation:C.M., P.S., and, A.B.; Writing — original draft preparation: C.M., P.S., and, A.B.; Writing — review and editing: C.M., and, H.C.; Visualization: C.M., P.S., A.B., and, H.C.; Supervision: H.C. All authors have read and agreed to the submitted version of the manuscript.

\section{ORCID Id:}

C.M.: 0000-0001-9324-7923; P.S.: 0000-0002-0184-9335; A.B.: 0000-0001-5919-1102; H.C.: 0000-0002-3925-4257

\section{Figure Caption:}

Figure 1. Temporal variation of ECG data: Comparative analysis of temporal variation of ECG time-series data of a normal subject (ID: 19090) (shown above) and an arrhythmic patient (ID: 100) (shown below).

Figure 2. Plots of IMFs retrieved following EMD Methodology: 12 IMFs were extracted by EMD performed on arrhythmic and normal ECG data. IMFs corresponding to one arrhythmic patient data (ID: 100) is shown.

Figure 3. Estimation of $\mathbf{H}$ of IMFs: $H$ values of an arrhythmic patient data (ID: 100) was calculated by means of $\mathrm{R} / \mathrm{S}$ technique.

Figure 4. Pattern recognition based on $\mathbf{H}$ of 1st IMFs: Distribution of $H$ values of 1 st IMFs of ECG data series represented by box and whisker plots demonstrated distinct patterns for arrhythmic (Disease) patients and normal controls.

Figure 5. Distinction in the $\mathbf{H}$ of 1st IMFs: The two-tailed student's t-test between the $H$ of all 1 st IMFs corresponding to the disease data and normal data.

Figure 6. Distinction in the $H$ of 1st IMFs based on age and gender: (A) A gender-based classification shows a variation in $\mathrm{H}$ of 1 st IMFs between arrhythmic (Disease) and normalECG signals between males and females. (B) An age-based subgrouping $(<30,30-50,50-70)$ also shows significant difference in $\mathrm{H}$ of 1 st IMFs between arrhythmic (Disease) and normal ECG signals.

\section{Table caption:}

Table 1: Comparison of $\mathrm{H}$ of different IMFs for a normal (Patient ID: 19090) and a diseased (Patient ID: 100) data

Table 2: Values of Hurst exponents of $1^{\text {st }}$ IMF of each (normal and arrhythmia disease) patients' data

Table 3: Statistical measures of $H$ of 1 st IMFs of the normal and arrhythmic (diseased) patients' ECG data according to age grouping 
Table 4: Statistical measures of $H$ of 1st IMFs of the normal and arrhythmic (diseased) patients' ECG data according to gender grouping

\section{References}

1. Hansson, A., Madsen-Härdig, B., \& Olsson, S. B. (2004). Arrhythmia-provoking factors and symptoms at the onset of paroxysmal atrial fibrillation: a study based on interviews with 100 patients seeking hospital assistance. $B M C$ cardiovascular disorders, 4(1), 13. https://doi.org/10.1186/1471-2261-4-13

2. Berkaya, S. K., Uysal, A. K., Gunal, E. S., Ergin, S., Gunal, S., \& Gulmezoglu, M. B. (2018). A survey on ECG analysis. Biomedical Signal Processing and Control, 43, 216-235. https://doi.org/10.1016/j.bspc.2018.03.003

3. Gupta, R., Guptha, S., Sharma, K. K., Gupta, A., \& Deedwania, P. (2012). Regional variations in cardiovascular risk factors in India: India heart watch. World journal of cardiology, 4(4), 112. $\underline{\text { https://doi.org/10.4330/wjc.v4.i4.112 }}$

4. de Godoy, M.F. (2016). Nonlinear analysis of heart rate variability: a comprehensivereview. Journalof Cardiology and Therapy, 3(3), 528-533. https://doi.org/10.17554/j.issn.2309-6861.2016.03.101-4

5. Agarwal, S., Rani, A., Singh, V., \& Mittal, A. P. (2016). Performance evaluation and implementation of FPGA based SGSF in smart diagnostic applications. Journal of medical systems, 40(3), 63. https://doi.org/10.1007/s10916-015-0404-2

6. Thakor, N. V., \& Zhu, Y. S. (1991). Applications of adaptive filtering to ECG a nalysis: noise cancellation and arrhythmia detection. IEEE transactions on biomedical engineering, 38(8), 785-794. https://doi.org/10.1109/10.83591

7. Poli, S., Barbaro, V., Bartolini, P., Calcagnini, G., \& Censi, F. (2003). Prediction of atrial fibrillation from surface ECG: review of methods and algorithms. Annali dell'Istituto superiore di sanità, 39(2), 195.

8. Korshunov, V., Penela, D., Linhart, M., Acosta, J., Martinez, M., Soto-Iglesias, D., ... \& Jáuregui, B. (2019). Prediction of premature ventricular complex origin in left vs. right ventricular outflow tract: a novel anatomical imaging approach. Ep Europace, 21(1), 147-153. https://doi.org/10.1093/europace/euy162

9. Shekatkar, S. M., Kotriwar, Y., Harikrishnan, K. P., \& Ambika, G. (2017). Detecting abnormality in heart dynamics from multifractal analysis of ECG signals. Scientific reports, 7(1), 1-11. https://doi.org/10.1038/s41598-017$\underline{15498-\mathrm{z}}$

10. Mohebbi, M., Ghassemian,H., \& Asl, B. M. (2011). Structures of the recurrence plot of heart rate variability signal as a tool for predicting the onset of paroxysmal atrial fibrillation. Journal of medical signals and sensors, 1(2), 113. https://doi.org/10.1016/j.cmpb.2015.12.008

11. Huang, N. E., Shen, Z., Long, S. R., Wu, M. C., Shih, H. H., Zheng, Q., ... \& Liu, H. H. (1998). The empirical mode decomposition and the Hilbert spectrum for nonlinear and non-stationary time series analysis. Proceedings of the Royal Societyof London. Series A: mathematical, physical and engineering sciences, 454(1971), 903-995. http://doi.org/10.1098/rspa.1998.0193

12. Blanco-Velasco, M., Weng, B., \& Barner, K. E. (2008). ECG signal denoising and baseline wander correction based on the empirical mode decomposition. Computers in biology and medicine, 38(1), 1-13. https://doi.org/10.1016/j.compbiomed.2007.06.003 
13. Tolwinski, S. (2007). The Hilbert Transform and Empirical Mode Decomposition as Tools for Data Analysis Real Signals and the Hilbert Transform. Transform, (1), 1-18.

14. Hurst, H. E. (1951). Long-term storage capacity of reservoirs. Transactions of the American Society of Civil Engineers, 116, 770-799.

15. Karegar, F. P., Fallah, A., \& Rashidi, S. (2017, May). ECG based human authentication with using Generalized Hurst Exponent. In 2017 Iranian Conference on Electrical Engineering (ICEE) (pp. 34-38). IEEE.

16. Moody, G. B., \& Mark, R. G. (2001). The impact of the MIT-BIH arrhythmia database. IEEE Engineering in Medicine and Biology Magazine, 20(3), 45-50. https://doi.org/10.1109/51.932724

17. Goldberger, A. L., Amaral, L. A., Glass, L., Hausdorff, J. M., Ivanov, P. C., Mark, R. G., ... \& Stanley, H. E. (2000). Current perspective. Circulation, 101, e215-e220. 10.1161/01.cir.101.23.e215

18. Hargittai, S. (2005, September). Savitzky-Golay least-squares polynomial filters in ECG signal processing. In Computers in Cardiology, 2005 (pp. 763-766). IEEE. https://doi.org/10.1109/CIC.2005.1588216

19. Savitzky, A., \& Golay, M. J. (1964). Smoothing and differentiation of data by simplified least squares procedures. Analytical chemistry, 36(8), 1627-1639. https://doi.org/10.1021/ac60214a047

20. Rilling, G., Flandrin, P., \& Goncalves, P. (2003, June). On empirical mode decomposition and its algorithms. In IEEE-EURASIP workshop on nonlinear signal and image processing (Vol. 3, No. 3, pp. 8-11). NSIP-03, Grado (I).

21. Ren, H., Wang, Y. L., Huang, M. Y., Chang, Y. L., \& Kao, H. M. (2014). Ensemble empirical mode decomposition parameters optimization for spectral distance measurement in hyperspectral remote sensing data. Remote Sensing, 6(3), 2069-2083. https://doi.org/10.3390/rs6032069

22. Barman, C., Ghose, D., Sinha, B., \& Deb, A. (2016). Detection of earthquake induced radon precursors by Hilbert Huang Transform. Journal of Applied Geophysics, 133, 123-131. https://doi.org/10.1016/j.jappgeo.2016.08.004

23. Peng, Z. K., Peter, W. T., \& Chu, F. L. (2005). A comparison study of improved Hilbert-Huang transform and wavelet transform: application to fault diagnosis for rolling bearing. Mechanical systems and signal processing, 19(5), 974-988. https://doi.org/10.1016/j.ymssp.2004.01.006

24. Wharton, A. M., Sekar Iyengar, A. N., \& Janaki, M. S. (2013). Study of nonlinear oscillations in a glow discharge plasma using empirical mode decomposition and Hilbert Huang transform. Physics of Plasmas, 20(2), 022301. https://doi.org/10.1063/1.4789853

25. Jha, R., Raju, D., \& Sen, A. (2006). Analysis of tokamak data using a novel Hilbert transform based technique. Physics of plasmas, 13(8), 082507. https://doi.org/10.1063/1.2218491

26. Mandelbrot, B. B., \& Wallis, J. R. (1969). Robustness of the rescaled range R/S in the measurement of noncyclic long run statistical dependence. Water resources research, 5(5), 967-988. https://doi.org/10.1029/WR005i005p00967

27. Hagiwara, Y., \& Faust, O. (2017). Nonlinear analy sis of coronary artery disease, myocardial infarction, and normal ECG signals. Journal of Mechanics in Medicine and Biology, 17(07), 1740006. doi:10.1142/s0219519417400061 
28. AlMahamdy, M., \& Riley, H. B. (2014). Performance study of different denoising methods for ECG signals. Procedia Computer Science, 37, 325-332. doi: 10.1016/j.procs.2014.08.048

29. Lastre-Dominguez, C., Shmaliy, Y. S., Ibarra-Manzano, O., Munoz-Minjares, J., \& Morales-Mendoza, L. J. (2019). ECG signal denoising and features extraction using unbiased FIR smoothing. BioMed research international, 2019. https://doi.org/10.1155/2019/2608547

30. Sahoo, S., Mohanty, M., Behera, S., \& Sabut, S. K. (2017). ECG beat classification using empirical mode decomposition and mixture of features. Journal of Medical Engineering \& Technology, 41(8), 652-661. https://doi.org/10.1080/03091902.2017.1394386

31. Looney, D., Hemakom, A., \& Mandic, D. P. (2015). Intrinsic multi-scale analysis: a multi-variate empirical mode decomposition framework. Proceedings of the Royal Society A: Mathematical, Physical and Engineering Sciences, 471(2173), 20140709. doi:10.1098/rspa.2014.0709

32. Xu, Y., Luo, M., Li, T., \& Song, G. (2017). ECG signal de-noising and baseline wander correction based on CEEMDAN and wavelet threshold. Sensors, 17(12), 2754. doi:10.3390/s17122754

33. Tavassoli, M., Ebadzadeh, M. M., \& Malek, H. (2012). Classification of cardiac arrhythmia with respect to ECG and HRV signal by genetic programming. Canadian Journal on Artificial Intelligence, Machine Learning and Pattern Recognition, 3(1), 1-8.

34. Karatoprak, E., \& Seker, S. (2019). An improved empirical mode decomposition method using variable window median filter for early fault detection in electric motors. Mathematical Problems in Engineering, 2019. doi:10.1155/2019/8015295

35. Draghici AE, Taylor JA (2016) The physiological basis and measurement of heart rate variability in humans. J Physiol Anthropol 35(1): 22. https://doi.org/10.1186/s40101-016-0113-7

36. John RM, Kumar S (2016) Sinus node and atrial arrhythmias. Contemporary Reviews in Cardiovascular Medicine 133:1892-1900. https://doi.org/10.1161/CIRCULATIONAHA.116.018011

37. Tse G (2016) Mechanisms of cardiac arrhythmias. Journal of Arrhythmia 32(2), 75-81. https://doi.org/10.1016/j.joa.2015.11.003

38. Subramanian B (2017) ECG signal classification and parameter estimation using multiwavelet transform. Biomed Res-India 28(7): 3187-3193.

39. Qian B, Rasheed K (2004) Hurst exponent and financial market predictability. Proceedings of the Second IASTED International Conference on Financial Engineering and Applications, 203-209.

40. Nayak, S. K., Bit, A., Dey, A., Mohapatra, B., \& Pal, K. (2018). A review on the no nlinear dynamical system analysis of electrocardiogram signal. Journal of healthcare engineering, 2018.

41. Luz, E. J. D. S., Schwartz, W. R., Cámara-Chávez, G., \& Menotti, D. (2016). ECG-based heartbeat classification for arrhythmia detection: A survey. Computer methods and programs in biomedicine, 127, 144-164.

42 Elhaj, F. A., Salim, N., Harris, A. R., Swee, T. T., \& Ahmed, T. (2016). Arrhythmia recognition and classification using combined linear and nonlinear features of ECG signals. Computer methods and programs in biomedicine, $127,52-63$. 
43. Haberl R, Jilge G, Pulter R, et al. Spectral mapping of the electrocardiogram with Fourier transform for identification of patients with sustained ventricular tachycardia and coronary artery disease. Eur Heart J 1989; 10:316-322.

44. Addison, P. S. (2005). Wavelet transforms and the ECG: a review. Physiological measurement, 26(5), R155. 

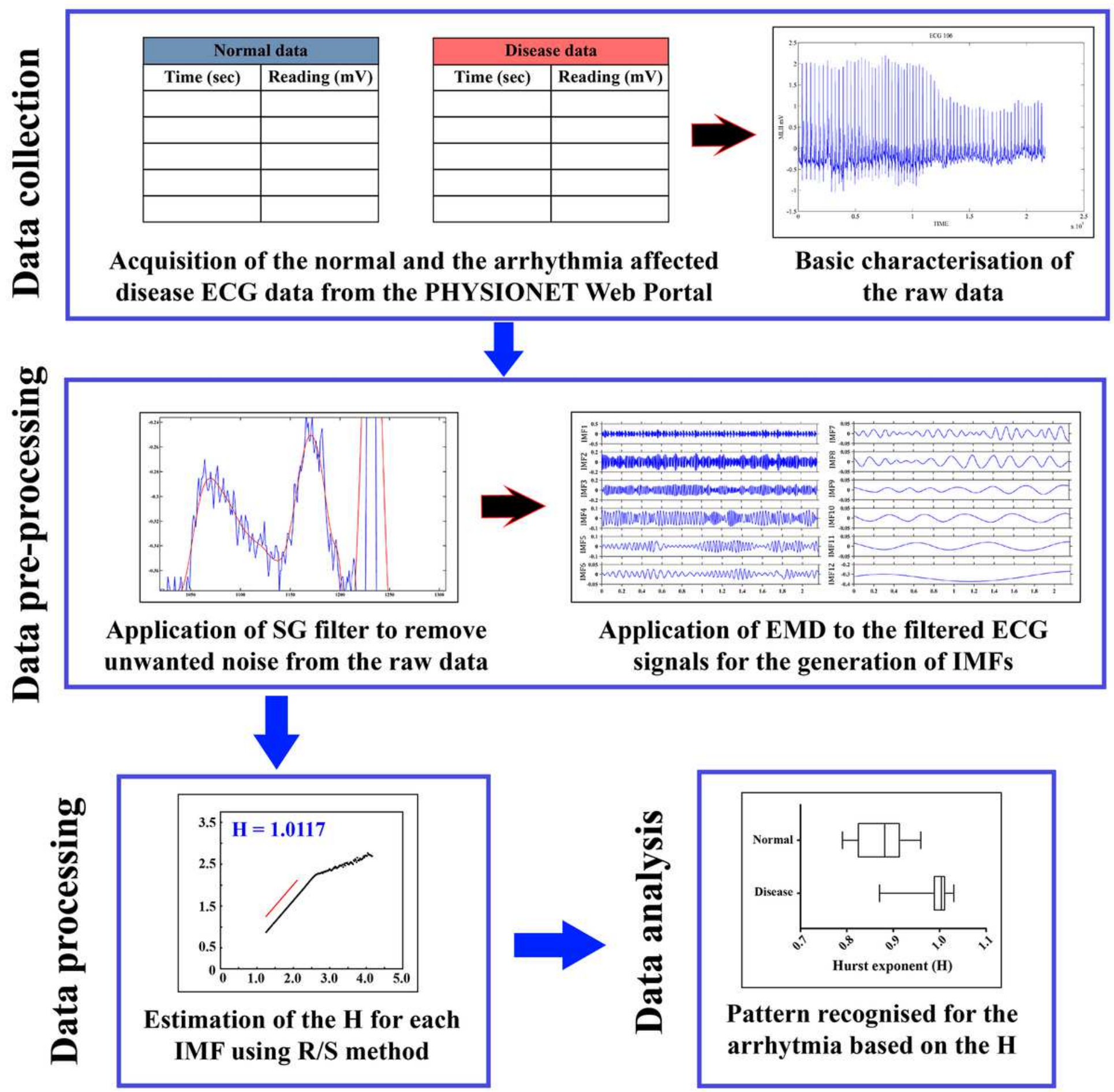

Figure 1

Temporal variation of ECG data: Comparative analysis of temporal variation of ECG time-series data of a normal subject (ID: 19090) (shown above) and an arrhythmic patient (ID: 100) (shown below). 


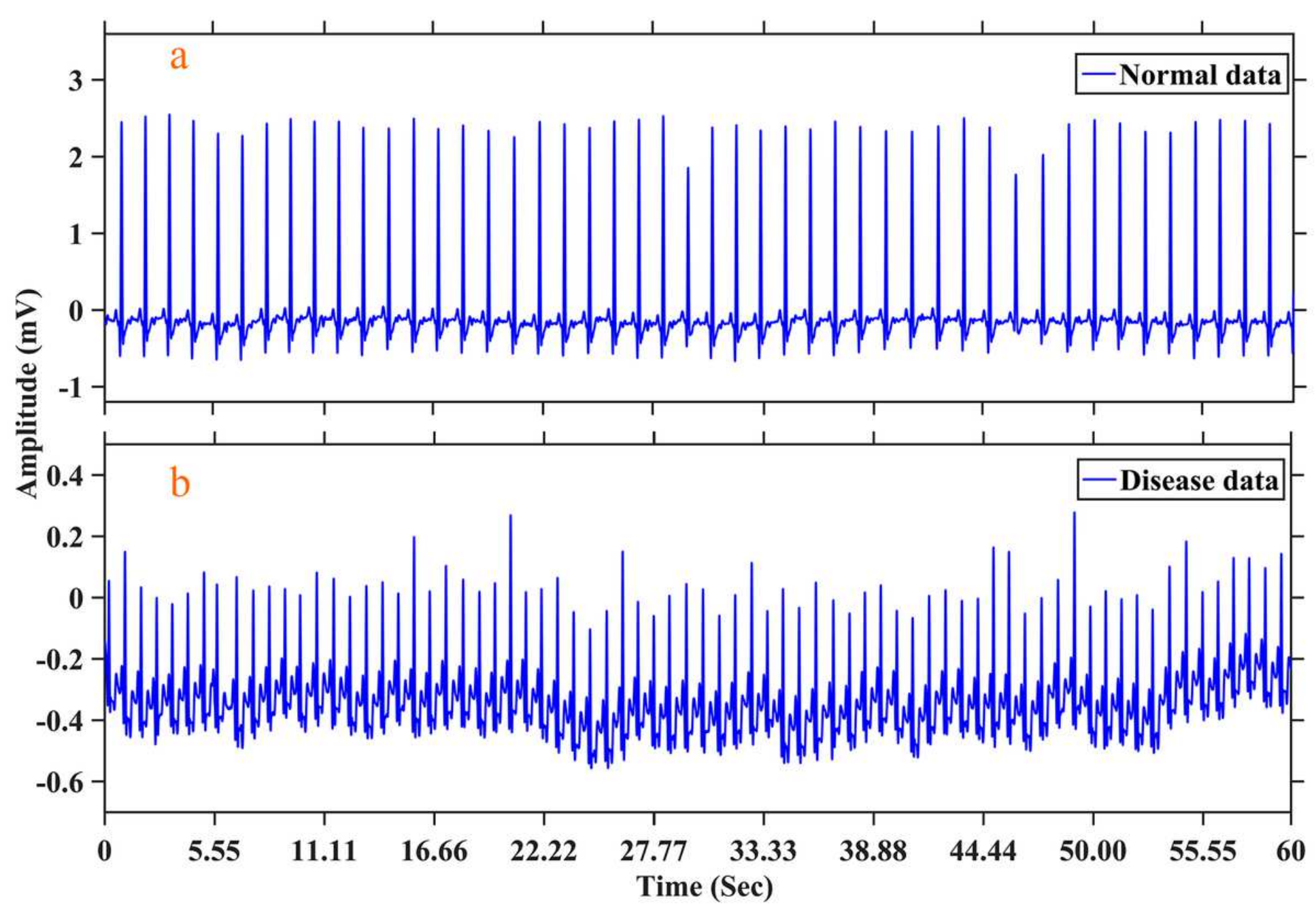

Figure 2

Plots of IMFs retrieved following EMD Methodology: 12 IMFs were extracted by EMD performed on arrhythmic and normal ECG data. IMFs corresponding to one arrhythmic patient data (ID: 100) is shown.
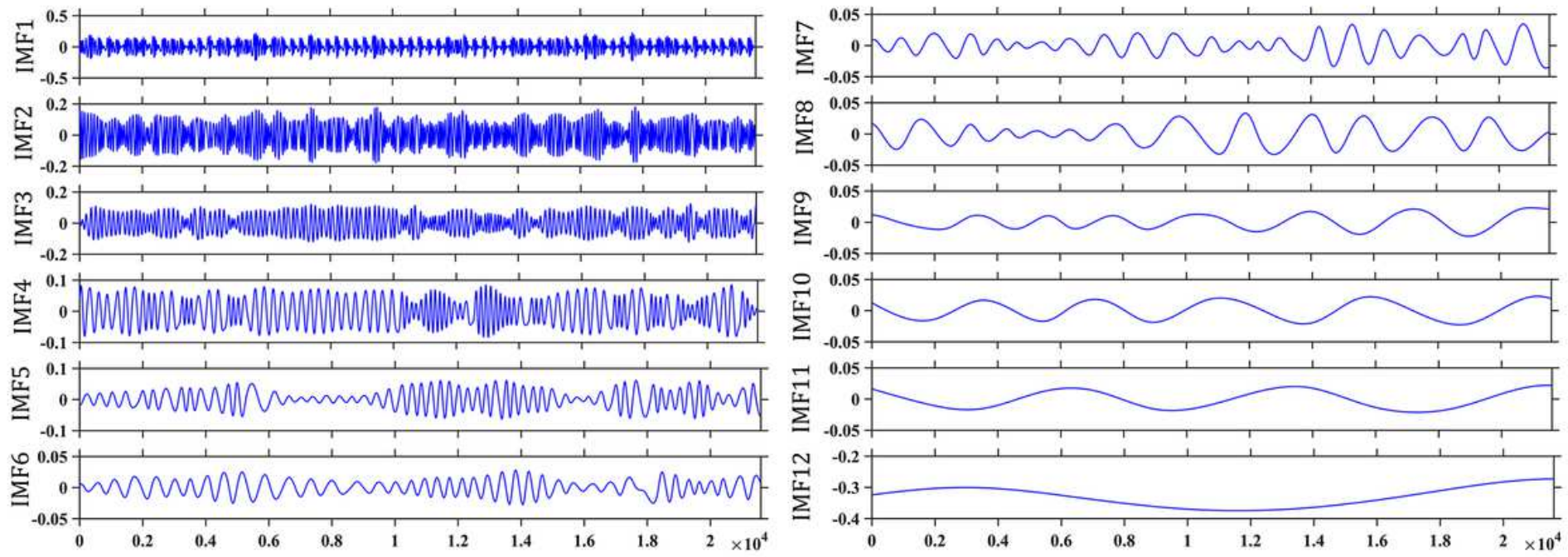

Figure 3 
Estimation of $\mathrm{H}$ of IMFs: $\mathrm{H}$ values of an arrhythmic patient data (ID: 100) was calculated by means of $\mathrm{R} / \mathrm{S}$ technique.

\section{R/S Method}

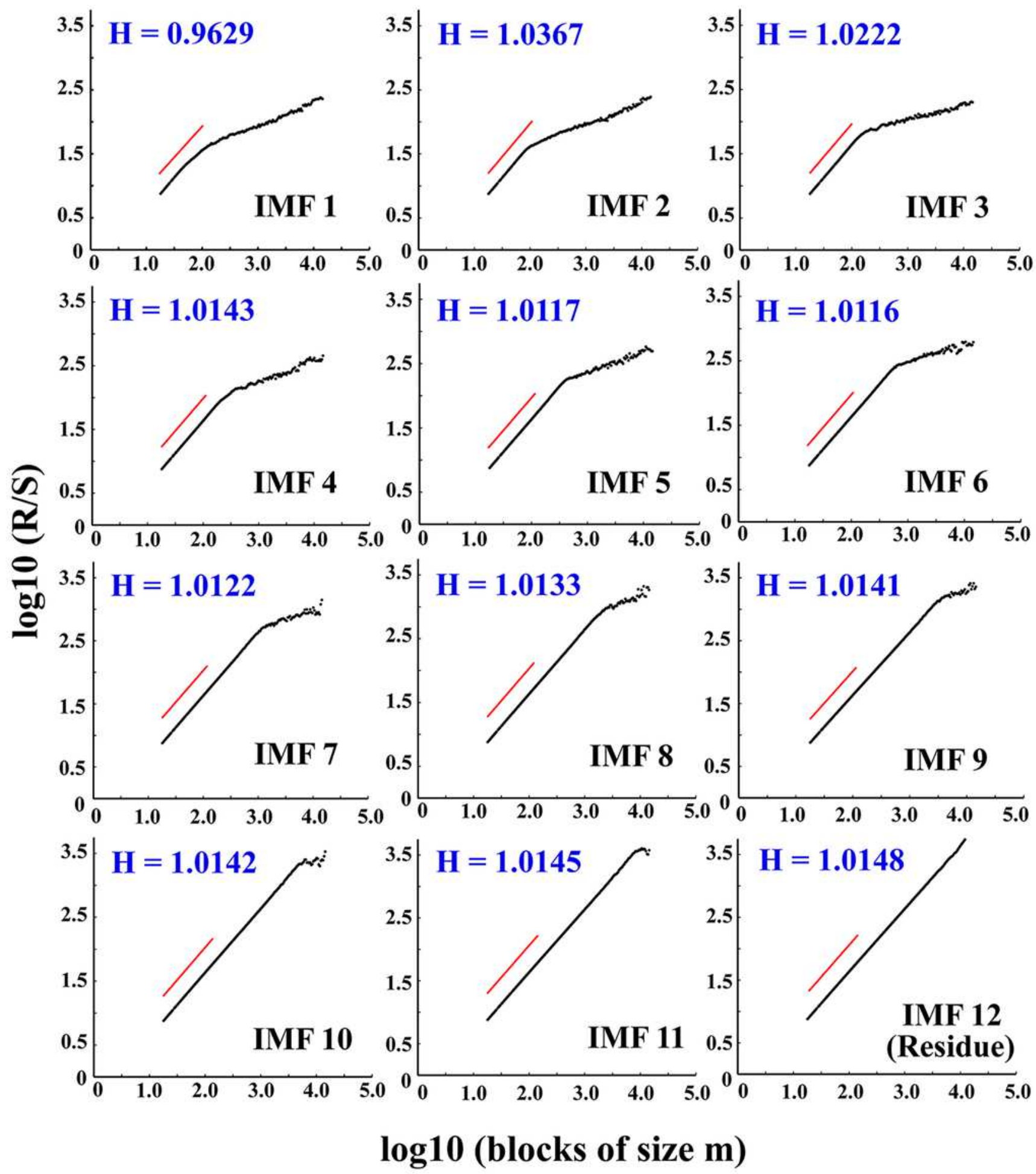

Figure 4

Pattern recognition based on $\mathrm{H}$ of 1 st IMFs: Distribution of $\mathrm{H}$ values of 1 st IMFs of ECG data series represented by box and whisker plots demonstrated distinct patterns for arrhythmic (Disease) patients and normal controls. 


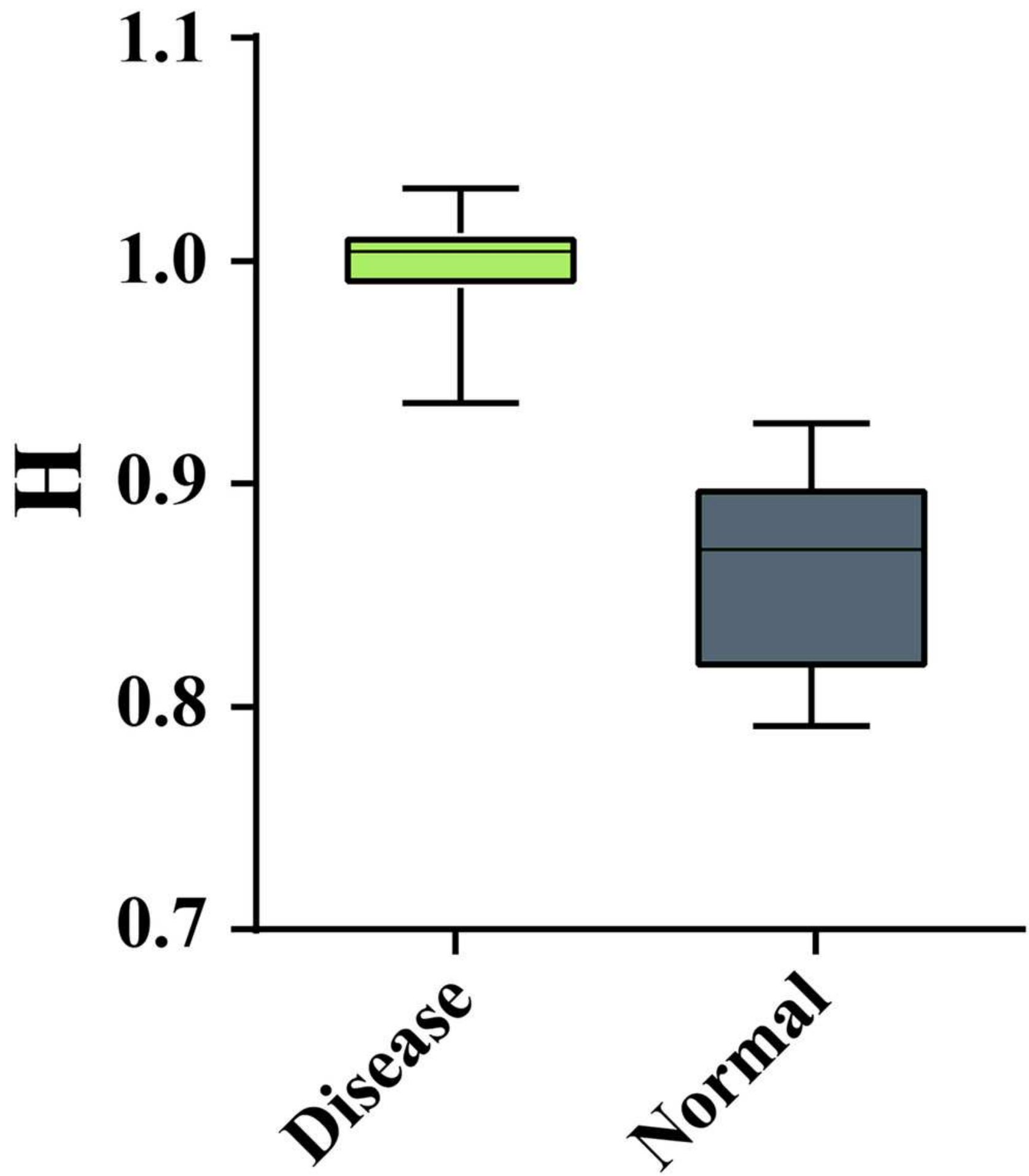

Figure 5

Distinction in the $\mathrm{H}$ of 1st IMFs: The two-tailed student's t-test between the $\mathrm{H}$ of all $1 \mathrm{st}$ IMFs corresponding to the disease data and normal data. 

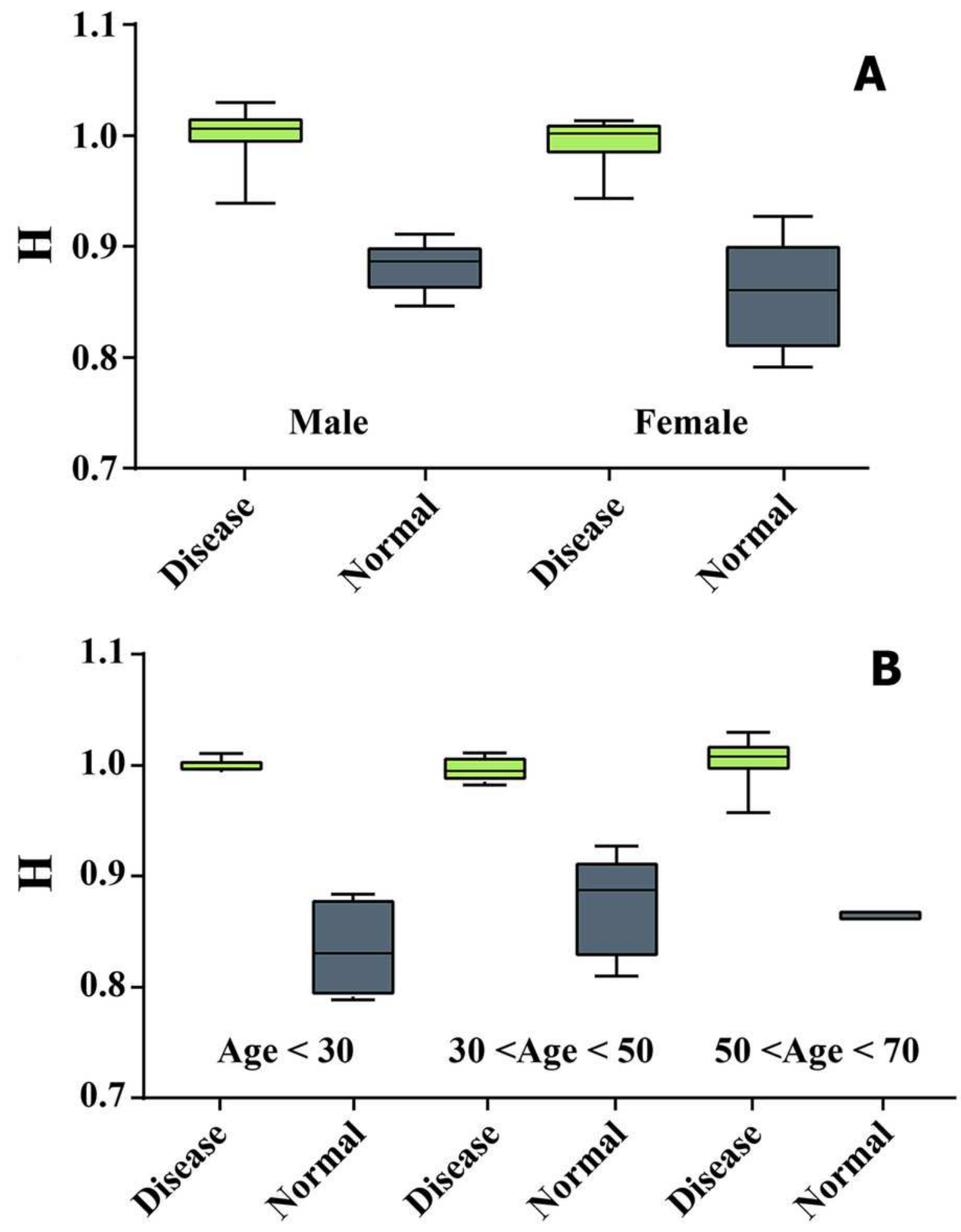

Figure 6

Distinction in the $\mathrm{H}$ of 1 st IMFs based on age and gender: (A) A gender-based classification shows a variation in $\mathrm{H}$ of $1 \mathrm{st}$ IMFs between arrhythmic (Disease) and normal ECG signals between males and females. (B) An age-based subgrouping (<30, 30-50,50-70) also shows significant difference in $\mathrm{H}$ of 1 st IMFs between arrhythmic (Disease) and normal ECG signals. 


\section{Supplementary Files}

This is a list of supplementary files associated with this preprint. Click to download.

- SupplementaryTableS1.xlsx

- SupplementaryTableS2.xlsx

- SupplementaryTableS3.xlsx 